\title{
An Inverse Relationship Between Weight and Free Thyroxine Middle Gestation
}

1,2Mariana Cristian Costache Outas, Cosmina-Raluca Costache ${ }^{3}$

1"Carol Davila" University of Medicine and Pharmacy, Bucharest, Romania; "'Coltea" Clinical Hospital, Bucharest, Romania; 3"Dr. Ioan Cantacuzino"'Clinical Hospital- Department of Obstetri0cs and Gynecology, Bucharest, Romania:

\section{OBJECTIVES}

Evaluation of thyroid function in pregnant women is extremely important for the health of mother and newborn. Reference range of thyroid function tests in pregnant women differs from that of the general population and between the trimesters in the same patient. Trimester-specific thyrotropin (TSH) reference ranges for pregnant women are recommended to avoid misclassification of the thyroid dysfunction during pregnancy. Less information about the range of free thyroxin (freeT4) specific-trimester is available. To test the variability of free thyroxin, we conducted a prospective pilot study.

\section{METHODS}

Forty-one consecutive pregnant women were selected for this study. They presented to the outpatient clinic referred by their obstetrician. We excluded patients with known hypothyroidism before pregnancy, patients with Basedow's disease and the patients in the third trimester of pregnancy (few cases).

We stratified the women into two groups according to TSH as having normal thyroid function, or subclinical hypothyroidism (SCH) using the reference interval for the first trimester, 0.1 to $2.5 \mathrm{mU} / 1$, and for the second trimester, 0.2 to $3.0 \mathrm{mU} / 1$.

The correlation of the parameters was performed using the Spearman correlation.

Comparison of median values between different groups was performed using the MannWhitney U test.

\section{RESULTS}

All patients come from an iodine sufficient area, but we did not test the level of urinary iodide. Table 1 shows the characteristics of patients, including TSH (in absolute value and expressed as a standard deviation score, SDS), free thyroxine, expressed as SDS, weight and number of the positive ATPO in each group. None of the euthyroid patients has positive

ATPO. 33.33\% of the pregnant women with SCH had positive ATPO.

Table 1 Characteristics of the patients.

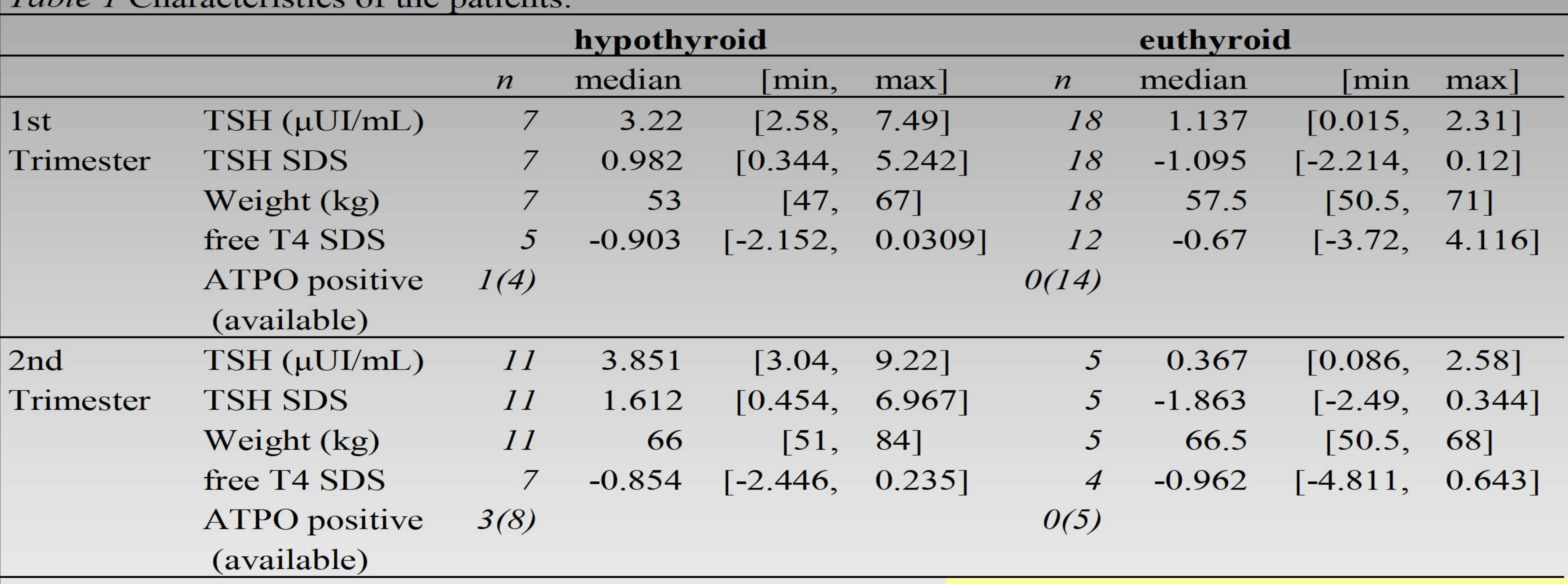

\section{CONCLUSIONS}

We found a negative correlation between the freeT4 SDS and TSH (rs= -.49; $\mathrm{P}=0.47$ ) in the first trimester of pregnancy, but not in the second.

Weight and TSH in ATPO positive patients was significantly higher compared to ATPO negative patients, $\mathrm{U}=29, \mathrm{z}=2.37, \mathrm{p}=0.014, \mathrm{r}=0.657$.

Weight negatively correlated with free T4 in hypothyroid patients but not in the euthyroid patients ( $\mathrm{rs}=-.657 ; \mathrm{P}=0.055$ and $\mathrm{rs}=-.003 ; \mathrm{P}=0.991$, respectively) in the 2nd trimester.

Mann-Whitney $U$ test revealed no significant differences for free thyroxine between first and second trimester of pregnancy.
We did not found differences in free T4 levels between first and second trimester of pregnancy. Free T4 levels were lower in mid-pregnancy as the pregnant women have a higher weight. ATPO antibody were positive exclusively in the state of subclinical hypothyroidism. Various reports about the variability of free thyroxine throughout pregnancy should take into account the patient's body weight, the iodine sufficiency, and the laboratory methodology.

\section{REFERENCES:}

. Lazarus, J. H. (2011). Thyroid function in pregnancy. British Medical Bulletin, 97(1), 137-148. doi:10.1093/bmb/ldq039

Stagnaro-Green, A., Abalovich, M., Alexander, E., Azizi, F., Mestman, J., Negro, R., ... Wiersinga, W. (2011). Guidelines of the American Thyroid Association for the Diagnosis and Management of Thyroid Disease During Pregnancy and Postpartum. Thyroid, 21(10), 1081-1125. doi:10.1089/thy.2011.0087 